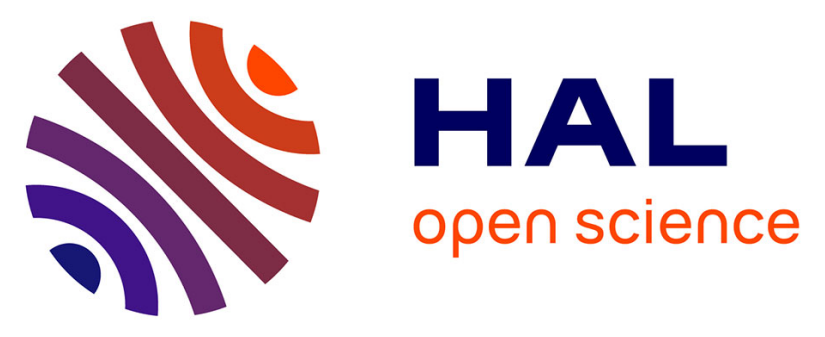

\title{
Statistical Characterization of Segregation Distance Among Cable Bundles Aboard Aircraft
}

Maria Denise Astorino, Giulio Antonini, A. Mori, P. Di Bartolomeo, M. Bandinelli, A. Bonsignore, Jean-Philippe Parmantier, Isabelle Junqua, Solange Bertuol, Jérome Morio, et al.

\section{To cite this version:}

Maria Denise Astorino, Giulio Antonini, A. Mori, P. Di Bartolomeo, M. Bandinelli, et al.. Statistical Characterization of Segregation Distance Among Cable Bundles Aboard Aircraft. 2020 International Symposium on Electromagnetic Compatibility - EMC EUROPE, Sep 2020, Rome (virtual), Italy. pp.1-6, 10.1109/EMCEUROPE48519.2020.9245756 . hal-03014678v2

\section{HAL Id: hal-03014678 \\ https://hal.science/hal-03014678v2}

Submitted on 4 Jun 2021

HAL is a multi-disciplinary open access archive for the deposit and dissemination of scientific research documents, whether they are published or not. The documents may come from teaching and research institutions in France or abroad, or from public or private research centers.
L'archive ouverte pluridisciplinaire HAL, est destinée au dépôt et à la diffusion de documents scientifiques de niveau recherche, publiés ou non, émanant des établissements d'enseignement et de recherche français ou étrangers, des laboratoires publics ou privés. 


\section{Statistical Characterization of Segregation Distance Among Cable Bundles Aboard Aircraft}

\author{
M. D. Astorino, G. Antonini \\ Università degli Studi dell'Aquila \\ L'Aquila, Italy \\ mariadenise.astorino@gmail.com \\ giulio.antonini@univaq.it,
}

\author{
A. Mori, P. di Bartolomeo, M. \\ Bandinelli, A. Bonsignore \\ I.D.S. Ingegneria dei Sistemi S.p.A. \\ Pisa, Italy \\ \{a.mori,p.dibartolomeo,m.bandinelli, \\ a.bonsignore\}@idscorporation.com
}

\author{
J. P. Parmantier, I. Junqua, \\ S. Bertuol, J. Morio \\ ONERA/DEMR Université de \\ Toulouse \\ Toulouse, France \\ $\{$ Jean-Philippe.Parmantier, \\ Isabelle.Junqua,Solange.Bertuol, \\ Jerome.Morio\}@onera.fr \\ C. Jullien \\ Safran Electrical \& Power \\ Blagnac, France \\ charles.jullien@safrangroup.com
}

\author{
N. Muot, C. Girard, G. Prin \\ AxesSim \\ Illkirch-Graffenstaden, France \\ \{nathanael.muot, christophe.girard, \\ guillaume.prin\}@axessim.fr
}

\begin{abstract}
Due to the large amount of electrical equipment aboard modern aircraft, several EMC problems appear which can be tackled by filtering disturbances at the equipment inputs and shielding cable links (braiding, covering, overbraiding). Among the others, the segregation between interfering cables or bundles of cables becomes crucial to ensure that coupling constraints will not exceed the equipment susceptibility threshold. The aim of this work is to statistically characterize the coupling between cable bundles and its minimization by optimization under stochastic constraints. The Monte Carlo method is used, and an interpolation scheme based on Smolyak grids is investigated to accelerate the computation.
\end{abstract}

Keywords-Cable bundles, Electric Wiring Interconnection System, Optimization, Statistical Methods.

\section{INTRODUCTION}

In order to limit EMC problems, the allocation of elementary cables in a bundle and installation of harnesses in structures must be controlled and must fulfil segregation rules given today by system integrators. Moreover, in aeronautics, the cabling, named EWIS for Electrical Wiring Interconnection System is now considered as a system as a whole which must follow specifications of the CS25-Subpart$\mathrm{H}$.

This is the reason why routes in which harnesses are gathered and run together in the structure are pre-defined in advance even if some parameters are unknown such as the cable length, the location of harnesses in the structure and the arrangement of harnesses. Therefore, depending on the type of useful signal and power carried on cables, distances of segregation are applied to ensure the inter-compatibility of routes. Nevertheless, sometimes, these rules of segregation cannot be fulfilled and distances between routes must be reduced. In this case, one must be able to evaluate the risk of this derogation in order to justify it and having it accepted.

In this context, Safran Electrical \& Power has initiated the CS2-ANALYST project (CFP07 GA 821128) with two Italian partners (IDS - Ingegneria Dei Sistemi and University of L'Aquila) and two French partners (AxesSim and ONERA). The objective of this project, as depicted in Fig. 1 is to implement a modeling tool [1] to evaluate within a reasonable time for a cable design team the optimum segregation distance, $d_{o p t}$, between two harnesses $\mathrm{A}$ and $\mathrm{B}$ connecting equipment, which will ensure the inter-compatibility considering the following inputs:

- Elementary cables in harnesses A and B;

- $\quad$ Input impedance of end-equipment $\left(Z_{A, N E, i} / Z_{A, F E, i}\right.$ and $\left.Z_{B, N E, i} / Z_{B, F E, i}\right)$;

- $\quad$ Sources generators $\left(V_{0, A, i} / I_{0, A, i}, V_{0, B, i} / I_{0, B, i}\right)$ at one extremity of harnesses;

- EM susceptibility thresholds $\left(S L_{A, i}\right.$ and $\left.S L_{B, i}\right)$ of equipment at opposite extremity in terms of voltages or currents.

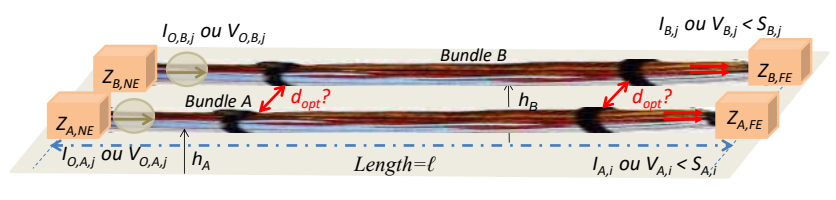

Fig. 1. EM problem.

The optimum segregation distance, $d_{o p t}$, must be valid for cable lengths $l$ (identical for both routes) and for heights of harnesses $h_{A}$ and $h_{B}$, varying in intervals specified by the enduser. This approach is conditioned by the fact that each harness, $\mathrm{A}$ or $\mathrm{B}$, have been previously correctly constituted in terms of auto-compatibility which means that inside a harness, sources will not generate levels of currents and voltages larger than the susceptibility thresholds of neighbouring cables.

Section II is dedicated to the mathematical formulation of the EM problem with the definition of the so-called " $\mathrm{G}$ criteria" considering the variation of input parameters and the resulting statistical analysis. The non-parametric technique for probability density estimation using Smolyak grids is described in Section III. This technique has been applied to a representative test case described in Section IV, followed by numerical results in Section V. Finally, Section VI gives some conclusions and future prospects of this work.

\section{MAthematicAl REPRESENTATION}

We have defined a mathematical elementary criteria $\mathrm{G}$, as a function of the segregation distance $d$, the coupling length $l$ and the heights of harnesses $h_{A}$ and $h_{B}$ as 


$$
G\left(d, l, h_{A}, h_{B}\right)=\max _{f, n A, n B}\left[\frac{o_{A, i}-S L_{A, i}}{S L_{A, i}}, \frac{o_{B, j}-S L_{B, j}}{S L_{B, j}}\right]
$$

where $O_{A, i}$ et $O_{B, j}$ are either voltages or currents, depending on the definition of susceptibility levels, on each elementary cable $i$ of harness $A$ and cable $j$ of harness $B$ induced by the source generators. The normalization of induced currents/voltages to the susceptibility levels $\left(S_{A, i}\right.$ and $\left.S_{B, i}\right)$ enable us to obtain a G-criteria which is dimensionless and therefore independent from the definition and nature of susceptibility thresholds as currents or voltages. From (1), if $\mathrm{G}$ is negative, then harnesses $A$ and $B$ are compatible, and their non-susceptibility is fulfilled.

Consequently, the solution to the initial EM problem consists in evaluating the smallest $d$-value, $d_{o p t}$, in order to fulfil (2):

$G\left(d_{\text {opt }} / l_{\min }<l<l_{\max } / h_{\min }<h_{A}, h_{B}<h_{\max }\right)<0$

Solving explicitly (2) is not trivial considering the complexity of harnesses in industrial cases as raised by Safran Electrical \& Power and the uncertainty of input parameters as the length and the heights over reference plane. This is the reason why a statistical approach has been preferred and proposed in this project. Nevertheless, the statistical analysis will give an optimum segregation distance under a targeted risk level (TRL). Therefore expression (2) can be replaced by:

$P\left\lfloor G\left(d_{\text {opt }} / l_{\min }<l<l_{\max } / h_{\min }<h_{A}, h_{B}<h_{\max }\right)<0\right\rfloor>T R L$

where $l, h_{A}$ and $h_{B}$ are uniform random variable between the specified minimal and maximal variation limits. The initial EM problem is now a problem of optimization under reliability constraints.

To this concern, Monte Carlo-based methods would guarantee high accuracy and ease of implementation. However, these methods entail the generation of a large number of random realizations of input variables and the consequent solution of deterministic simulations for each realization. Thus, they are sampling-based approaches, widely used for uncertainty propagation studies in aerospace engineering; they make few assumptions about the system but are usually computationally expensive because of the large number of realizations needed to achieve statistical convergence.

An efficient alternative is represented by stochastic spectral methods such as polynomial chaos (PC) expansion and stochastic collocation methods based on Smolyak quadrature, based on sparse grids that allow efficient representation and interpolation functions on multidimensional hypercubes [2]. Such a method is used in the following section and has been applied to a representative test case for validation versus the traditional Monte Carlo method.

\section{NONPARAMETRIC TECHNIQUE FOR PROBABILITY DENSITY ESTIMATION}

One way to evaluate the Smolyak interpolation results and to provide the probability that the induced currents satisfy the susceptibility level is to compare the Smolyak cumulative

Identify applicable funding agency here. If none, delete this text box. distribution functions (CDF) with the reference CDF computed on the Full grid.

In general, the data set ( $\mathrm{G}$ parameters or current/voltage outputs) is supposed to be a sample, statistically significant, with an unknown probability density function (PDF). The data set is employed to estimate the PDF rather than deciding in advance if the PDF belongs to a given parametric family by following a nonparametric approach [3], [4]. Specifically, the density histograms and the kernel density estimates (KDE) have been applied as statistical methods. Since the PDF/CDF computed with the two nonparametric techniques have provided similar results, in what follows, we have reported only the probability functions computed through the KDE method.

The basic idea of KDE is to estimate the density function at a point $X_{i}$ using neighbouring observations. Kernel density estimates are closely related to histograms but can be endowed with properties such as smoothness or continuity by using a suitable kernel function. Hence, instead of building up the estimate according to bin edges, the kernel method adaptively uses each point of estimation as the centre of the bin.

A kernel estimator can be expressed by:

$$
\hat{f}_{\text {Ker }}(x)=\frac{1}{n h} \sum_{i=1}^{n} K\left(\frac{x-X_{i}}{h}\right)
$$

where $n$ is the number of data points, $x$ is the set of samples (domain), and $h$ is the smoothing parameter. The estimated PDF $\hat{f}_{\text {Ker }}(x)$ is obtained through a weighted kernel function centred at each data point and then by taking the average of them. Moreover, $\hat{f}_{\text {Ker }}(x)$ inherits all of the properties of the kernel function, such as continuity and differentiability, being the estimator an additive function of the kernel weight. Due to its convenient mathematical properties, the normal kernel has been adopted in the current analysis in order to obtain a smooth PDF with derivatives of all orders:

$$
K(x)=\frac{1}{\sqrt{2 \pi}} e^{-1 / 2^{x^{2}}}
$$

The $h$ parameter, also called "window width", determines the amount of smoothing in the estimate $\hat{f}_{\text {Ker }}(x)$, a characteristic shared by all nonparametric curve estimators [5]. An optimal window width can be obtained by minimizing the asymptotic mean integrated squared error (AMISE) and by applying the normal reference rule:

$$
h_{\text {Ker }}^{*}=\left(\frac{4}{3}\right)^{1 / 5} \sigma n^{-1 / 5}
$$

where $\sigma$ is the median absolute deviation estimator $\sigma=$ median $\left|X_{i}-\mu_{m}\right| / 0.6745$ with $\mu_{m}$ the median of the sample, in order to accommodate long-tailed distributions and possible outliers.

Once the PDF is estimated, the CDF can be computed by integrating under the PDF curve, from which every cumulative distribution function is non-decreasing and rightcontinuous.

\section{BUNDLE PAIR UNDER STUDY}

A well-controlled test case has been identified in order to assess our statistical approach. A bundle pair composed of 12 conductors in the first bundle (Bundle A) and 24 conductors 
in the second bundle (Bundle B) has been considered as a test case for the following statistical analyses. All the 36 conductors are parallel to each other with different radii ranging from 0.72 to $2.15 \mathrm{~mm}$ and are surrounded by dielectric coatings with relative permittivity $\varepsilon_{r}=2.3$. The two bundles are located above an infinite perfect electric conductor (PEC) plane, as shown in Fig. 2(a).

The geometrical configuration of the bundle pair is described by means of four parameters: the bundle length $l$ in the range [10-70] $\mathrm{m}$, the distance $d$ between the centres of the bundles [0.02-0.50] $\mathrm{m}$, and the height of the first (second) bundle $h_{A}\left(h_{B}\right)$ [0.01-0.20] $\mathrm{m}$ from the bundle canter to the PEC plane.

Fig. 2(b) describes the source terms and terminal loads applied to each conductor of the bundle pair. As shown in the circuital representation, all conductors of both bundles are loaded by a common mode resistance of $9 \Omega$ at the input/output extremities, while a voltage generator of $115 \mathrm{~V}$, constant over the frequency range from $f_{\min }=10 \mathrm{kHz}$ to $f_{\text {max }}=$ $200 \mathrm{MHz}$, is applied on all 12 input ports of the first bundle.

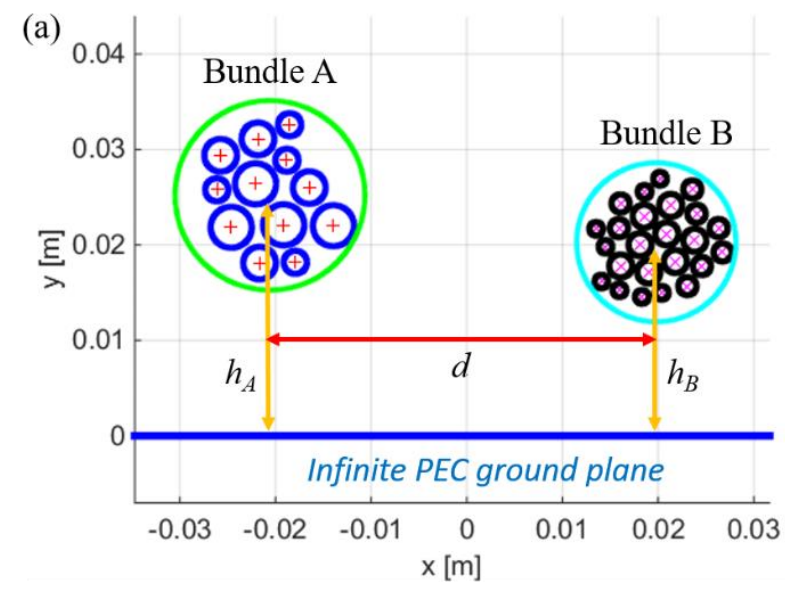

(b)

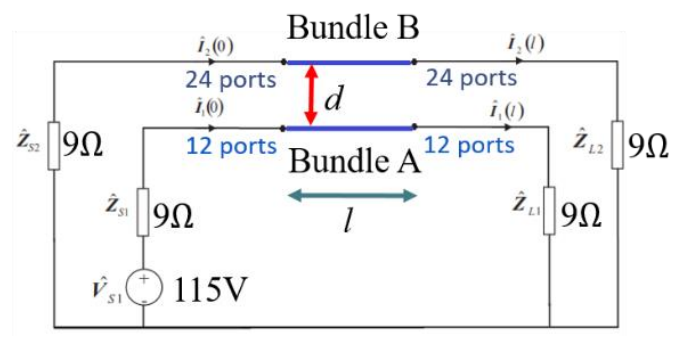

Fig. 2. (a) Bundle pair 2D geometrical cross-section and (b) source terms and terminal loads.

In order to solve the associated multiconductor transmission lines (MTL) problem, the per-unit length (p.u.l.) electrical parameters (resistance $R$, inductance $L$, and capacitance $C$ ) have been computed with the Laplace module that is a sub-module of the CRIPTE code [8].

As stated in the introduction, the objective of our statistical analysis is to determine the optimized segregation distance $d_{\text {opt }}$ in order to guarantee that the currents induced on each conductor of the second bundle do not overcome the susceptibility level set as $\mathrm{SL}=1.5 \mathrm{~A}$ in the frequency range of interest. In order to satisfy this constraint, the induced currents have been computed through CRIPTE (MTL Network solver developed by ONERA [8]) simulations for a given set of variable parameters $\left(l, h_{A}, h_{B}\right)$ and given segregation distances $d=4 ; 10 ; 15 ; 30 \mathrm{~cm}$.

Along with the induced currents, the G-parameter values are obtained through the function as given by (1).

In what follows, we have considered two configuration sets: the first validation set, named "Full grid", is composed of 1000 geometrical configurations $\left(l, h_{A}, h_{B}\right)$ for each given segregation distance, while the second training set, named "Smolyak grid", is used to interpolate the simulation outputs ( $\mathrm{G}$ parameters or induced currents) on multidimensional hypercubes of the Full grid.

From the CRIPTE calculation and the subsequent $G$ parameters calculation, the Smolyak interpolation method [2] can be performed either directly on the G-function (later on called "Smolyak G") or on the current outputs $\mathrm{I}_{\mathrm{w}}$ (later on called "Smolyak I") computed on the Smolyak grid.

In the latter case, the interpolated parameters $G_{\text {int }}$ for each geometric configuration of the Full grid have been retrieved by applying the $\mathrm{G}$ definition in (1) and by substituting the maxima of the interpolated induced currents $\mathrm{I}_{\mathrm{int}, \mathrm{w}}$ for each wire.

\section{NUMERICAL RESULTS}

Since the electromagnetic compatibility problem in our analysis involves large-scale systems, the Smolyak method has been selected, as it was not subject to the curse of dimensionality. However, although the Smolyak method allows studying high dimensional problems more efficiently than other methods such as tensor-product or multidimensional quadrature rules, its computational cost still grows rapidly with the dimensionality of the problem. In order to overcome this limitation, we have considered a more effective implementation of the Smolyak method involving the use of disjoint-set generators rather than the conventional nested-set generators [2]. This allowed avoiding costly evaluations of the repeated basis functions particularly burdensome in the case of these high-dimensional problems. Indeed, the conventional Smolyak formula computes a long list of repeated basis functions and constructs linear combinations of such functions to get rid of repetitions for interpolating a function at a given point. The disjoint-set generators reduce this computational expense since they do not introduce repetitions in the tensor products.

Moreover, while the conventional Smolyak method is isotropic, namely, the same number of grid points and basis functions is used for all variables in a symmetric way, it is also possible to implement an anisotropic variant of Smolyak method. This is useful to separately set the accuracy level for each dimension in order to increase the quality of the approximation. The asymmetric treatment of variables turns out to be particularly effective when some variables have larger ranges of values or when some variables are more sensitive than others. In our EMC problem, since it is not trivial to establish a priori the most sensitive variables and after conducting several attempts with anisotropic Smolyak grids, we have adopted isotropic Smolyak grids in order to consider the same accuracy level $\mu$ for all the geometric parameters. 
As a first example, we have supposed a reduced length range between 10 and $15 \mathrm{~m}$ in order to limit current fluctuations, and an approximation parameter $\mu=5$, obtaining a Smolyak grid composed of 1105 geometrical configurations for the four parameters $l ; d ; h_{A} ; h_{B}$. The results in Fig. 3 represent the comparison between the $\mathrm{PDF} / \mathrm{CDF}$ of the interpolated solutions either starting from the $G$ parameters (Smolyak G) or frm the current outputs $\mathrm{I}_{\mathrm{w}}$ (Smolyak I) of the CRIPTE solver, together with the rigorous solutions at given segregation distances $d=4 ; 10 ; 15 ; 30 \mathrm{~cm}$. Table I summarizes the probability values that the susceptibility level of $1.5 \mathrm{~A}$ is satisfied, by comparing the two Smolyak G/I interpolations with the reference probabilities computed on the 1000-point Full grid.
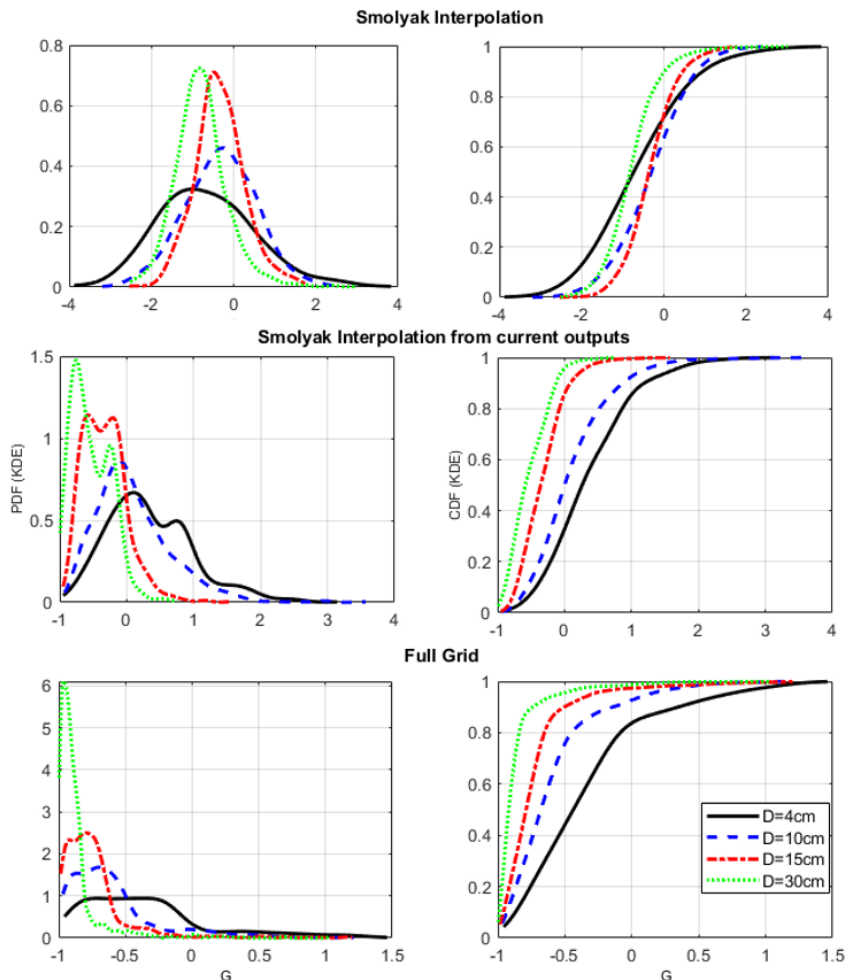

ull Grid

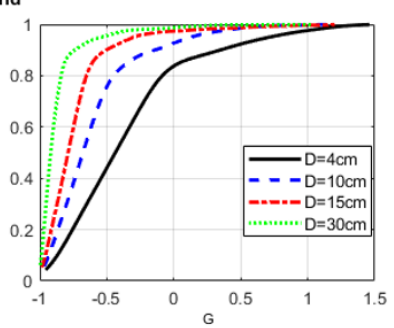

Fig. 3. PDF and CDF of the interpolated Smolyak G, Smolyak I, and 1000point Full grid solutions (from the top to bottom) with $\mathrm{d}=4 ; 10 ; 15 ; 30 \mathrm{~cm}$ and bundle length range $l[10-15] \mathrm{m}$ for 1105-point Smolyak grid.

TABLE I. PRoBABILITY TO SATISFY THE SUSCEPTIBILITY LEVEL $\mathrm{SL}=1.5$ A FOR 1105-POINT SMOLYAK GRID AND 1000-POINT FULL GRID

\begin{tabular}{|c|c|c|c|}
\hline \multirow{2}{*}{$\begin{array}{c}\boldsymbol{d} \\
{[\mathbf{c m}]}\end{array}$} & \multicolumn{3}{|c|}{$\mathbf{P}\left[\mathbf{G}\left(\boldsymbol{d} ; \boldsymbol{h}_{\boldsymbol{A}} ; \boldsymbol{h}_{\boldsymbol{B}} ; \boldsymbol{l} \boldsymbol{i} \mathbf{S L}\right)<\mathbf{0}\right]$} \\
\cline { 2 - 4 } & Smolyak $\boldsymbol{G}$ & Smolyak $\boldsymbol{I}$ & Full Grid \\
\hline 4 & $72.11 \%$ & $32.42 \%$ & $83.70 \%$ \\
\hline 10 & $63.31 \%$ & $49.38 \%$ & $92.72 \%$ \\
\hline 15 & $72.68 \%$ & $85.48 \%$ & $97.36 \%$ \\
\hline 30 & $89.63 \%$ & $95.87 \%$ & $98.69 \%$ \\
\hline
\end{tabular}

In general, it can be observed that the probability to overcome the susceptibility level SL increases when the segregation distance D decreases. Intuitively, this is due to the higher electrical coupling between bundles when the distance is progressively reduced.

However, the comparison of the Smolyak approach with the Full grid shows some discrepancies in the evaluation of the probability $\mathrm{P}[\mathrm{G}<0]$, especially at the lowest distances, as shown in Tab. I. In order to investigate this behaviour, we have to consider that the induced currents $I_{w}$ show a smooth low-frequency trend from $10 \mathrm{kHz}$ to about $5 \mathrm{MHz}$, while at higher frequencies $(5-200 \mathrm{MHz})$ they resonate generating a set of multiple resonances/anti-resonances. In our analysis, we have computed only $60 \mathrm{log}$-spaced frequencies between 10 $\mathrm{kHz}$ and $200 \mathrm{MHz}$; in the domain of resonances and zeros the current is thereby under-sampled. Consequently, the Gfunction strongly depends on the frequency sampling and on the bundle lengths. This is why the G-function shows abrupt irregularities even in the case of geometrical configurations very similar from a practical perspective, i.e. for parameter variations of the order of $1 \mathrm{~mm}$.

As an example, Fig. 4 illustrates the currents versus frequency computed by CRIPTE on an elementary wire of harness $\mathrm{B}$ for two different but very close geometrical configurations in terms of length (difference of $10 \mathrm{~cm}$ ) and heights (difference of $5 \mathrm{~mm}$ ) over the reference plane. Considering the definition of the $\mathrm{G}$-criteria, $\mathrm{G}$ is maximum for two different frequencies and its value differs from a factor of 10 which shows strong irregularities of the G-criteria.

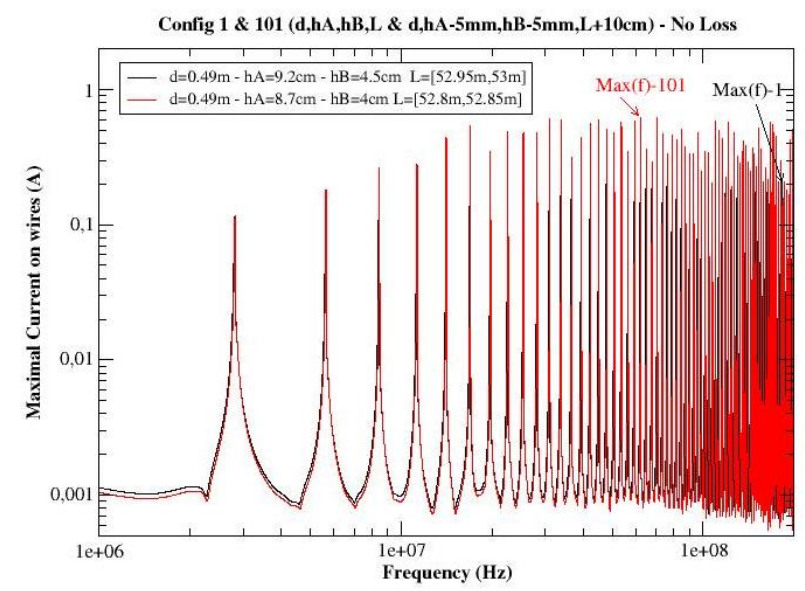

Fig. 4. CRIPTE calculations of currents onto a wire of bundle B for two very close geometrical configurations.

Therefore, the Smolyak interpolation technique applied to these irregular G-functions is less efficient and requires an enrichment of the grid points at the expense of the computational resources.

As a result, we have improved the Smolyak grid by setting the approximation parameter $\mu=6$ and further reducing the distance range $D$ between 4 and $30 \mathrm{~cm}$. The resulting Smolyak grid is composed of 2929 different combinations of the four parameters $l ; d ; h_{A} ; h_{B}$. For each of these configurations, the CRIPTE solver provides the induced currents on the second bundle from which the $\mathrm{G}$ parameters are post-processed and the relevant Smolyak interpolating coefficients are computed. In this way, the true function and its approximation coincide in all Smolyak grid points, and the retrieved coefficients can be substituted in the Smolyak formula using unidimensional disjoint sets to interpolate the solution on the 1000-point Full grid. 
Fig. 5 shows the PDF/CDF of the interpolated Smolyak G and Smolyak I solutions, while Tab. II reports the probability values that the $G$ parameter is less than zero, i.e., the probability that the $\mathrm{SL}=1.5 \mathrm{~A}$ is satisfied, in comparison with the reference Full grid results (see Fig. 3) computed for specific segregation distances.
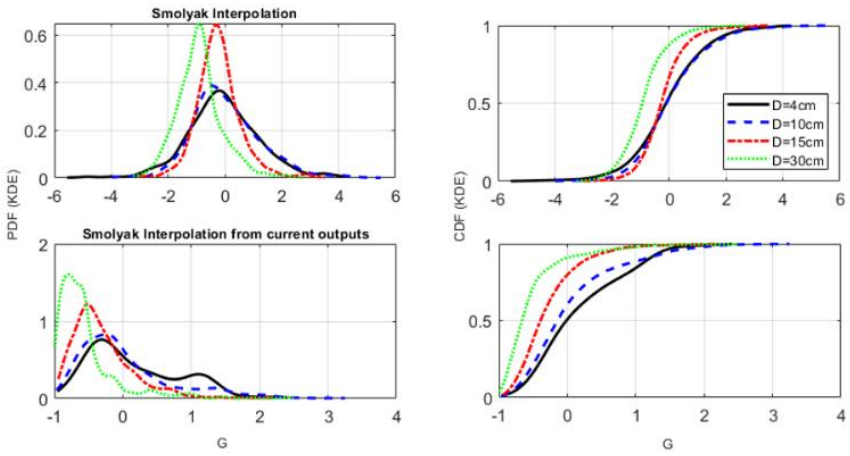

Fig. 5. PDF and CDF of the interpolated Smolyak G (top) and Smolyak I (bottom) solutions with $\mathrm{d}=4 ; 10 ; 15 ; 30 \mathrm{~cm}$ and bundle length range $l$ [1015] $\mathrm{m}$ for 2929-point Smolyak grid.

TABLE II. PROBABILITY TO SATISFY THE SUSCEPTIBILITY LEVEL $\mathrm{SL}=1.5$ A FOR 2929-POINT SMOLYAK GRID AND 1000-POINT FULL GRID.

\begin{tabular}{|c|c|c|c|}
\hline \multirow{2}{*}{$\begin{array}{c}\boldsymbol{d} \\
{[\mathbf{c m}]}\end{array}$} & \multicolumn{3}{|c|}{$\mathbf{P}\left[\mathbf{G}\left(\boldsymbol{d} ; \boldsymbol{h}_{\boldsymbol{A}} ; \boldsymbol{h}_{\boldsymbol{B}} ; \boldsymbol{l}\right.\right.$; SL $\left.)<\mathbf{0}\right]$} \\
\cline { 2 - 4 } & Smolyak $\boldsymbol{G}$ & Smolyak I & Full Grid \\
\hline 4 & $53.41 \%$ & $50.85 \%$ & $83.70 \%$ \\
\hline 10 & $52.75 \%$ & $60.67 \%$ & $92.72 \%$ \\
\hline 15 & $65.39 \%$ & $80.05 \%$ & $97.36 \%$ \\
\hline 30 & $87.59 \%$ & $91.10 \%$ & $98.69 \%$ \\
\hline
\end{tabular}

In order to investigate the effectiveness of the Smolyak technique for interpolating highly irregular G-functions, we have further enriched the Smolyak grid points by setting an approximation level $\mu=7$ for all geometrical parameters. The obtained isotropic Smolyak grid is composed of 7537 configurations and it has been employed to interpolate both $\mathrm{G}$ parameters and induced currents on the Full grid. This has allowed us to estimate the PDF and CDF of the G-function with an increased level of accuracy with respect to the 2929points Smolyak grid, as shown in Fig. 6. This is even more evident from $\mathrm{Tab}$. III that compares the probability values $\mathrm{P}[\mathrm{G}$ $<0]$ computed through Smolyak methods with the reference Full grid.
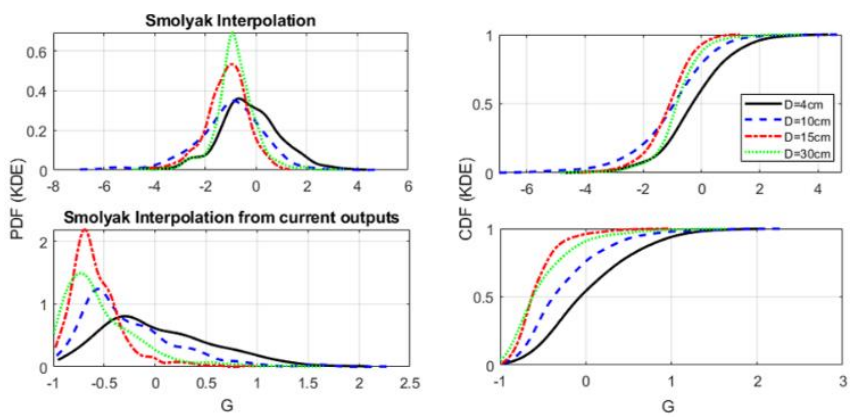

Fig. 6. PDF and CDF of the interpolated Smolyak G (top) and Smolyak I (bottom) solutions with $\mathrm{d}=4 ; 10 ; 15 ; 30 \mathrm{~cm}$ and bundle length range $l$ [1015] $\mathrm{m}$ for 7537-point Smolyak grid.
TABLE III. PRoBABILITy To SATISFy ThE SUSCEPTIBILITy LEVEL $\mathrm{SL}=1.5$ A FOR 7537-POINT SMOLYAK GRID AND 1000-POINT FULL GRID.

\begin{tabular}{|c|c|c|c|}
\hline \multirow{2}{*}{$\begin{array}{c}\boldsymbol{d} \\
{[\mathbf{c m}]}\end{array}$} & \multicolumn{3}{|c|}{$\mathbf{P}\left[\mathbf{G}\left(\boldsymbol{d} ; \boldsymbol{h}_{\boldsymbol{A}} ; \boldsymbol{h}_{\boldsymbol{B}} ; \boldsymbol{l} ; \mathbf{S L}\right)<\mathbf{0}\right]$} \\
\cline { 2 - 4 } & Smolyak $\boldsymbol{G}$ & Smolyak $\boldsymbol{I}$ & $\boldsymbol{F u l l}$ Grid \\
\hline 4 & $60.48 \%$ & $54.46 \%$ & $83.70 \%$ \\
\hline 10 & $78.82 \%$ & $75.87 \%$ & $92.72 \%$ \\
\hline 15 & $92.59 \%$ & $95.96 \%$ & $97.36 \%$ \\
\hline 30 & $87.80 \%$ & $90.58 \%$ & $98.69 \%$ \\
\hline
\end{tabular}

As a further example, we have considered an ideal bundle pair with the same geometry and dimensions as in Fig. 2, but without dielectric coatings around the 36 conductors. In this case, analytical formulas of p.u.l. parameters are available ([6], [7]):

$$
\begin{gathered}
R=\operatorname{diag}\left(\frac{1}{\sigma \pi r^{2}}\right) \\
L_{a}=\frac{\mu_{0}}{2 \pi} \log \left(\frac{2 h_{w}}{r}\right) \\
L_{m}=\frac{\mu_{0}}{4 \pi} \log \left(1+\frac{4 h_{w 1} h_{w 2}}{d_{m}^{2}}\right) \\
C=\mu_{0} \varepsilon_{0} L^{-1}
\end{gathered}
$$

where $\sigma=10^{7} \mathrm{~S} / \mathrm{m}$ is the electrical conductivity, $r$ is the radius of each conductor, $L_{a}$ is the self-inductance, $h_{w}$ is the height of each conductor from the ground plane, $L_{m}$ is the mutual inductance, $h_{w 1}\left(h_{w 2}\right)$ is the height of each conductor of the first (second) harness from the PEC plane, $d_{m}$ is the mutual distance between the conductors, $\mu_{0}$ is the vacuum permeability, and $\varepsilon_{0}$ is the vacuum permittivity. These p.u.l . parameters contain all information about the cross-sectional dimensions of the bundle pair under test.

For this analysis, the range of variation for the distance is between 4 and $30 \mathrm{~cm}$, while for the bundle length is from 10 to $15 \mathrm{~m}$. The accuracy level was set equal to five for all the parameters for the construction of a 1105-point isotropic Smolyak grid. Fig. 7 compares the PDF/CDF of the interpolated solutions of the CRIPTE solver starting from the $G$ parameters (Smolyak $G$ ) and the induced currents $I_{w}$ (Smolyak I) with the rigorous solutions of a 1000-point Full grid computed for each given segregation distance. As it can be observed from Tab. IV, the lack of dielectric coatings increases the probability that the susceptibility level is satisfied and for the segregation distance $\mathrm{D}=30 \mathrm{~cm}$ all the induced currents are below the $\mathrm{SL}=1.5 \mathrm{~A}$ limit. In this case, the Smolyak interpolation technique is able to better reproduce the probability estimates, since the G-functions are subject to fewer variations in the frequency range. 

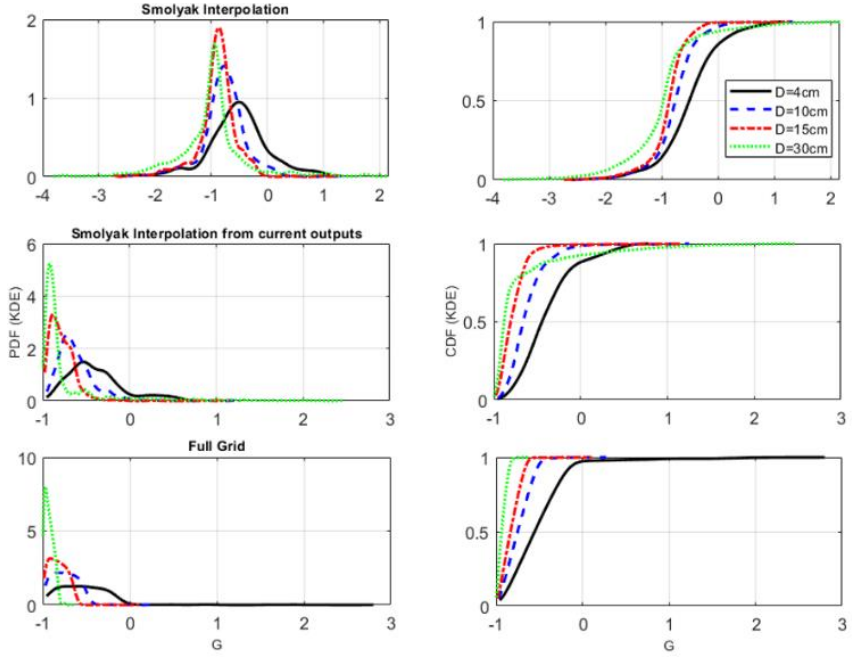

Fig. 7. PDF and CDF of the interpolated Smolyak G, Smolyak I, and 1000 point Full grid solutions (from the top to bottom) with $\mathrm{d}=4 ; 10 ; 15 ; 30 \mathrm{~cm}$ and bundle length range $l[10-15] \mathrm{m}$ for 1105-point Smolyak grid on an ideal bundle pair.

TABLE IV. Probability To SATISFy The SusceptibiLity LEVEL $\mathrm{SL}=1.5$ A FOR 1105-POINT SMOLYAK GRID AND 1000-POINT FULL GRID ON AN IDEAL BUNDLE PAIR.

\begin{tabular}{|c|c|c|c|}
\hline \multirow{2}{*}{$\begin{array}{c}\boldsymbol{d} \\
{[\mathbf{c m}]}\end{array}$} & \multicolumn{3}{|c|}{$\mathrm{P}\left[\mathbf{G}\left(\boldsymbol{d} ; \boldsymbol{h}_{\boldsymbol{A}} ; \boldsymbol{h}_{\boldsymbol{B}} ; \boldsymbol{l} ; \mathbf{S L}\right)<\mathbf{0}\right]$} \\
\cline { 2 - 4 } & Smolyak $\boldsymbol{G}$ & Smolyak I & Full Grid \\
\hline 4 & $85.70 \%$ & $88.07 \%$ & $97.23 \%$ \\
\hline 10 & $96.79 \%$ & $98.75 \%$ & $99.86 \%$ \\
\hline 15 & $99.38 \%$ & $99.39 \%$ & $99.90 \%$ \\
\hline 30 & $93.88 \%$ & $92.67 \%$ & $100.00 \%$ \\
\hline
\end{tabular}

In general, from Figs. 3, 5-7 and the relevant Tabs. I-IV, it can be observed that the Smolyak solutions obtained from the induced currents (Smolyak I) provide PDF/CDF shapes and probability values for $\mathrm{P}[\mathrm{G}<0]$ more similar to the rigorous solutions than the Smolyak solutions obtained from the $G$ parameters (Smolyak G). This is due to the larger information content of the complex values of the induced currents compared to that of the real values of the $G$ parameters.

\section{CONCLUSIONS}

In this work, we have investigated the EMC problem of segregation distance between cable-harnesses installed in aircraft and its optimization under stochastic constraints. To this end, the Smolyak interpolation technique has been applied in the analysis of real and ideal bundle pairs with variable installation parameters and given termination loads. The $\mathrm{G}$ parameters and induced currents outputs of an MTL model implemented in the CRIPTE solver have been interpolated on multidimensional hypercubes of the Full grid at fixed distances by means of isotropic disjoint-set Smolyak grids. For the data post-processing, we have selected nonparametric techniques to compute the probability density and cumulative distribution functions. In particular, the kernel density estimation has been employed by choosing an optimal window width able to reduce the error measurement on both the Full and Smolyak grids. In order to test the effectiveness of the Smolyak interpolation method, we have conducted several tests by increasing the approximation levels $\mu$. These denser Smolyak grids have actually improved the interpolated solutions for this high-dimensional problem, but the intrinsic irregularity of the G-function over the wide intervals of variation of geometrical parameters and frequencies makes the figure of merit not trivial to interpolate. Overall, the Smolyak I solutions have provided a better estimate of the reference solutions thanks to the larger information content of the induced current complex values $I_{w}$.

\section{ACKNOWLEDGMENT}

This project has received funding from the Clean Sky 2 Joint Undertaking under the European Union's Horizon 2020 research and innovation programme under grant agreement No 821128.

\section{REFERENCES}

[1] A. Mori, P. di Bartolomeo, M. Bandinelli, A. Bonsignore, G. Antonini, M. D. Astorino, N. Muot, C. Girard, G. Prin, J. P. Parmantier, I. Junqua, S. Bertuol, J. Morio, C. Jullien," Design-oriented EMC analysis of wiring systems", EMC-Europe 2020 Proceedings. To be published.

[2] K. L. Judd, L. Maliar, S. Maliar and R. Valero, "Smolyak method for solving dynamic economic models: Lagrange interpolation, anisotropic grid and adaptive domain," Journal of Economic Dynamics \& Control, vol. 44, pp. 92-123, 2014.

[3] D. W. Scott, Multivariate density estimation: theory, practice, and visualization, John Wiley \& Sons, 2015.

[4] D. Poljak, S. Šesnić, M. Cvetković, A. Šušnjara, H. Dodig, S. Lalléchère and K. Drissi, "Stochastic Collocation Applications in Computational Electromagnetics," Mathematical Problems in Engineering, vol. 2018, pp. 1-13, 2018.

[5] A. W. Bowman and A. Azzalini, Applied smoothing techniques for data analysis: the kernel approach with S-Plus illustrations. Vol. 18. OUP Oxford, 1997.

[6] C. R. Paul, Electromagnetic compatibility. Encyclopedia of RF and Microwave Engineering, 2005.

[7] C. R. Paul, Analysis of Multiconductor Transmission Lines. New York: Wiley, 1992.

[8] J. P. Parmantier, S. Bertuol, and I. Junqua, "CRIPTE : Code de réseaux de lignes de transmission multiconducteur - User's Guide - Version 5.1” ONERA/DEMR/T-N119/10 - CRIPTE 5.12010. 